\title{
Ovarian Cancer and Primary Peritoneal Carcinoma pNX TNM Finding v7
}

National Cancer Institute

\section{Source}

National Cancer Institute. Ovarian Cancer and Primary Peritoneal Carcinoma pNX TNM

Finding v7. NCl Thesaurus. Code C89655.

Ovarian cancer or primary peritoneal carcinoma in which regional lymph nodes cannot be assessed. (from AJCC 7th Ed.) 